The Free Internet Journal for Organic Chemistry
Paper

Arkivoc2019, part v, 0-0

to be inserted by editorial office

\title{
Green and efficient synthesis of 1,2-bis(2H-benzo[e][1,3]oxazin-3(4H)-yl)ethanes and 1,2-bis(2H-benzo[e][1,3]thiazin-3(4H)-yl)ethanes
}

\author{
R.L. Nongkhlaw, ${ }^{a}$ R. Nongrum, ${ }^{a}$ S. Tumtin, ${ }^{b}$ and I. T. Phucho ${ }^{b *}$ \\ ${ }^{a}$ Department of Chemistry \\ North Eastern Hill University, Shillong-793022, Meghalaya, India \\ ${ }^{b}$ Department of Chemistry \\ Nagaland University, Lumami-798627, Nagaland, India \\ *Email: itphucho@gmail.com
}

Received 12-18-2018

Accepted 04-26-2019

Published on line 05-19-2019

\section{Abstract}

An environmental friendly procedure for synthesis of 1,2-bis $(2 H$-benzo[e][1,3]oxazin-3(4H)-yl)ethanes and 1,2bis $(2 H$-benzo[ $e][1,3]$ thizin-3 $(4 H)$-yl)ethanes is reported using water as solvent. A comparative study between a conventional and a microwave method is also reported.

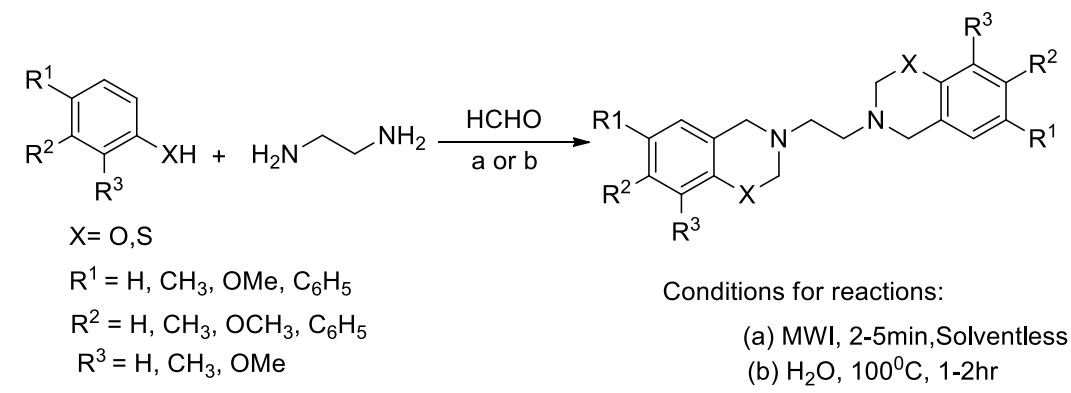

Keywords: Oxazines, benzoxazines, microwave, green chemistry 


\section{Introduction}

Heterocycles containing the oxazine nucleus possess a wide range of biological applications. ${ }^{1}$ Of the many reported pathways, the Mannich reaction involving phenols, formalin and primary amines ${ }^{2}$ is advantageous for developing a variety of heterocyclic compounds owing to its convenience and ease of reagent availabilty. The interest for 1,3-oxazines has recently increased, mainly due to compounds containing a dihydro-1,3-oxazine ring system leading to a wide spectrum of pharmacological activities such as antitumor, ${ }^{3}$ anti-bacterial, ${ }^{4}$ anti-HIV5and anti-malarial agents ${ }^{6}$ and their versatility as synthetic intermediates. ${ }^{7}$ In addition, naphthoxazine derivatives exhibit therapeutic potential for the treatment of Parkinson's disease. ${ }^{8}$

Despite the fact that substituted 1,3-thiazines have been known for more than a century and constitute the structural basis of several biologically active substances of both natural and synthetic origin, methods for the synthesis of these heterocyclic systems have not been well developed. Various benzothiazine derivatives are known to possess a versatile range of biological activities and have been synthesized continuously since the very first synthesis by Abe et al. ${ }^{9}$ Among these, 1,2-benzothiazine-3-carboxamide-1,1dioxides such as piroxicam, ${ }^{10}$ ampiroxicam ${ }^{11}$ and meloxicam ${ }^{12}$ are familiar for their analgesic and antiinflammatory activities and are being used worldwide as non-steroidal anti-inflammatory drugs (NSAIDs).

A literature survey revealed that when one biodynamic heterocyclic system was coupled with another, a molecule with enhanced biological activity ${ }^{13}$ was produced. The chemistry of these bi-heterocycles has afforded a fascinating investigation in the field of medicinal chemistry as they have been found to exhibit an enhanced biological profile. ${ }^{14}$ Synthesis and investigation of the activity of compounds in which an isoxazole moiety is linked with a benzoxazine nucleus have been reported by Reddy et al. ${ }^{15}$ in a study on the antimicrobial activity of 3-(3,5-dimethyl-isoxazol-4-yl)-3,4-dihydro-2H-benzo[e][1,3]-oxazines.

\section{Synthesis of bisoxazines and bisthiazines}

Our main focus in this paper is on bis-1,3-oxazines and bis-1,3-thiazines. Thorough literature survey revealed that the compounds containing a dihydro-1,3-oxazine ring system exhibited a wide spectrum of pharmacological activity and ever since the first isolation of 2, 4-dihydroxy-2H-1, 4-benzoxazin-3(4H)-one (DIBOA) and 4-dihydroxy-7-methoxy-(2H)-1,4-benzoxazin-3(4H)-one (DIMBOA), benzoxazine derivatives have attracted the attention of phytochemists. ${ }^{16}$

Our literature survey revealed that the synthesis of bis-heterocyclic compounds has become an important field of research for finding new biologically active molecules.

Bisheterocyclic compounds possess important pesticidal properties, ${ }^{17}$ anti-bacterial properties ${ }^{18}$ and anti-tumor activity. ${ }^{19}$ Following these reports, we envisaged that molecules with two benzoxazine/benzothiazine rings linked through flexible aliphatic chains or through rigid aromatic chains could have enhanced biological activities.

P. R. Carlier and co-workers ${ }^{20}$ worked on the synthesis and evaluation of alkylene-linked dimers of tacarine (9-amino-1,2,3,4-tetrahydroacridine). The reaction of tacarine with dibromo alkanes gave the desired bis-product,but in low yields (epecially when $n=2$ to $n=6$ ). To overcome this difficulty, Carlier and co-workers explored the reaction of 9-chloro-1,2,3,4-tetrahydroacridine with diamines. This method was found to be successful and the optimum conditions proved to be in refluxing 1-pentanol at atmospheric pressure for 40 hours.

To enhance the thermal characteristics, Ninan et $a{ }^{21}{ }^{21}$ synthesized a bis-benzoxazine monomer with additional polymerizable allyl groups substituted on the active ortho sites of bisphenol- $A$. The composition, 
structure, cyclic ratio and polymer structure of a diallyl di-benzoxazine prepared by a suspension method were studied. ${ }^{22}$

Information on bisbenzoxazines is scarce, but this interesting class of compounds acts as key precursors that leads to nitrogen-containing heterocycles as well as skeletons of biologically related molecules.

Bisbenzoxazines exhibit various biological activities including antibacterial, antitumor and plant growth regulative properties. Bis-benzoxazine monomers containing phenylphosphine oxide have been synthesized from phosphorous containing bisphenol compounds, primary amine and formaldehyde. ${ }^{23}$

The present paper describes a synthetic study of a series of bis-oxazine and bis-thiazine derivatives, two oxazine/thiazine monomers connected by aliphatic alkyl linkers through an eco-friendly Mannich type condensation-cyclization reaction of phenols or naphthol with formaldehyde and primary amines in water under reflux or under microwave irradiation.

Synthetic approaches to 3,4-dihydro-2H-1,3-benzothiazines include (i) condensation of 4,5-dimethoxy2-mercaptobenzylammonium chloride with an aromatic aldehyde in the presence of potassium carbonate ${ }^{24}$ and (ii) cycloaddition of benzothiete with the corresponding substituted imines. ${ }^{25}$

The synthesis of oxazine and thiazine monomers has been reported extensively, but bis-oxazines have not been well documented. The synthesis of two new bis-benzoxazines via Mannich reaction of $\beta$ - and $\alpha$-naphthol with formaldehyde and ethylendiamine was also reported by Augusto Rivera and co-workers. ${ }^{26} \mathrm{R}$. Manikannan and S. Muthusubramanian ${ }^{23}$ have successfully described the synthesis of symmetrical bis-benzoxazines using microwave irradiation as well as the possibility of a multicomponent approach for the synthesis of the target molecule.

\section{Results and Discussion}

Dihydro-1,3-oxazines have been reported to have a strong cytotoxic effect on tumor cells, especially inat low concentrations. Reports on the synthesis of 3,4-dihydro-2H-1,3-benzoxazines describe (i) Mannich condensation of phenol and a primary amine with formaldehyde, ${ }^{27}$ (ii) condensation of $o$-hydroxybenzylamine with an aldehyde, ${ }^{28}$ (iii) rearrangement reactions of 2-(allyloxy)benzylamine with $\mathrm{H}_{2} / \mathrm{CO}$ in the presence of rhodium catalysts, ${ }^{29}$ (iv) condensation of a 4-substituted phenol with 1,3,5-trimethyl-hexahydro-triazine in the presence of oxalyl chloride, ${ }^{30}$ (v) reaction of 1 -(bromomethyl)-2 (chloromethoxy)benzene with primary amines, $^{31}$ (vi) dehydration of $\mathrm{N}$-(2-hydroxybenzyl)-3-aminopropanoic acid in the presence of sulfuric acid ${ }^{32}$ and (vii) by ortho lithiation. ${ }^{35}$

The synthesis of various 3,4-dihydro- $2 H$-benzo[e]-2,3-dihydro- $1 H$-naphtho[1,2-e]-, 3,4-dihydro- $2 \mathrm{H}$ naphtho[2,1-e][1,3]oxazines and 1,2-bis[3,4-dihydrobenzo[e][1,3]oxazin-3(4H)-yl]ethanes involves a one-pot condensation-cyclization reaction of phenols or naphthol with formaldehyde and primary amines. Various methods have been reported ${ }^{34-45}$ for the synthesis of dihydro-1,3-oxazines including the reaction under neat conditions. ${ }^{46}$ However, many of these processes have disadvantages such as the need for a prolonged reaction time, high temperature, use of volatile and toxic organic solvents and the presence of side products.

In view of these liabilities, this paper presents a green synthesis of a new heterocyclic compounds with a benzoxazine unit. With a vast number of suitable starting compounds available, multifunctional amine-based bisbenzoxazines and bis-benzothiazines have tremendous untapped potential in the area of tailoring molecular structure for specific applications. A solvent-less reaction is typically the synthetic procedure of choice for the preparation of benzoxazine monomers, primarily due to simplicity and purity of the products. 
However, the high reactivity of the linear aliphatic diamines produced an elevated concentration of oligomeric species during the solvent-less protocol. In an effort to minimize the generation of oligomers, the synthesis was conducted with the reactants diluted in water $(2 \mathrm{ml} / \mathrm{mmol})$. A variety of bisoxazines and bis-thiazines having aliphatic linkages between the two monomers have been synthesized, under green chemistry conditions. (Scheme-1 and Scheme-2).

The structures of the linear aliphatic diamine-based benzoxazines were confirmed by NMR, FTIR and mass spectropmetry. Each ${ }^{1} \mathrm{H}$ NMR spectrum showed two singlet peaks, centered at approximately 3.9 and 4.8 ppm, consistent with the formation of a benzoxazine ring. The $-\mathrm{CH}_{2}$ - peak appears around 2.9 ppm as a singlet. The Mannich bridge protons of the open oxazine rings are typically ${ }^{47}$ located at approximately 3.7 . The absence of any peaks in this region indicates that the alcohol washes were successful in separating the oligomeric species from the monomers. Integration shows the closed-ring content of each compound to be higher than $98 \%$. For polymerization kinetic studies, high purity of the synthesized compounds is of utmost importance since the phenolic impurity acts as the cationic initiator for benzoxazine polymerization and thus adversely affects the precision of the study.

As expected, the infrared spectra of the benzoxazine monomers are nearly identical for all of the different diamine chain lengths. There are a number of infrared bands in the spectra, which can be used to verify the formation of oxazine rings in each product. While not shown, the presence of the benzoxazine ring aromatic ether is confirmed using absorbance peaks at 1043 and $1208 \mathrm{~cm}^{-1}$, due to the C-O-C symmetric and the asymmetric stretching modes, respectively. ${ }^{48}$ Absorbance at $771 \mathrm{~cm}^{-1}$ shows the presence of the expected ortho-substituted benzene rings, while the peaks at 857 and $930 \mathrm{~cm}^{-1}$ confirms the presence of benzene with an attached oxazine ring. Also noteworthy is the complete lack of bands from free or hydrogen-bonded hydroxyl groups. The absence of hydroxyl groups shows that the reaction optimization and purification has successfully eliminated any unreacted phenol and oligomeric species.

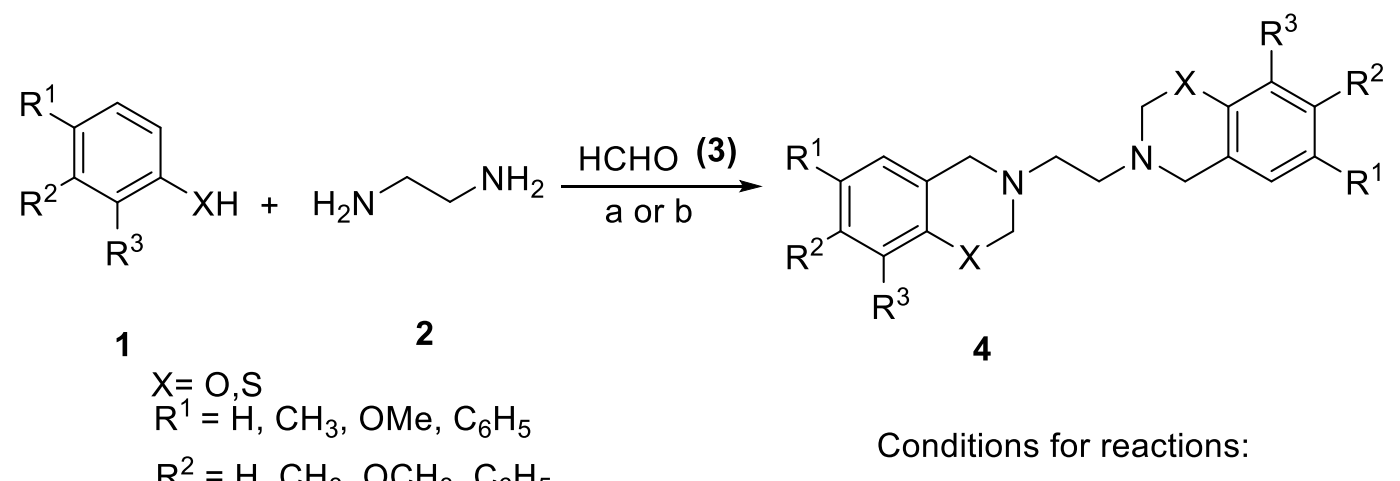

$\mathrm{R}^{2}=\mathrm{H}, \mathrm{CH}_{3}, \mathrm{OCH}_{3}, \mathrm{C}_{6} \mathrm{H}_{5}$
$\mathrm{R}^{3}=\mathrm{H}, \mathrm{CH}_{3}, \mathrm{OMe}$

(a) MWI, 2-5min, Solventless

(b) $\mathrm{H}_{2} \mathrm{O}, 100^{\circ} \mathrm{C}, 1-2 \mathrm{hr}$

\section{Scheme 1}




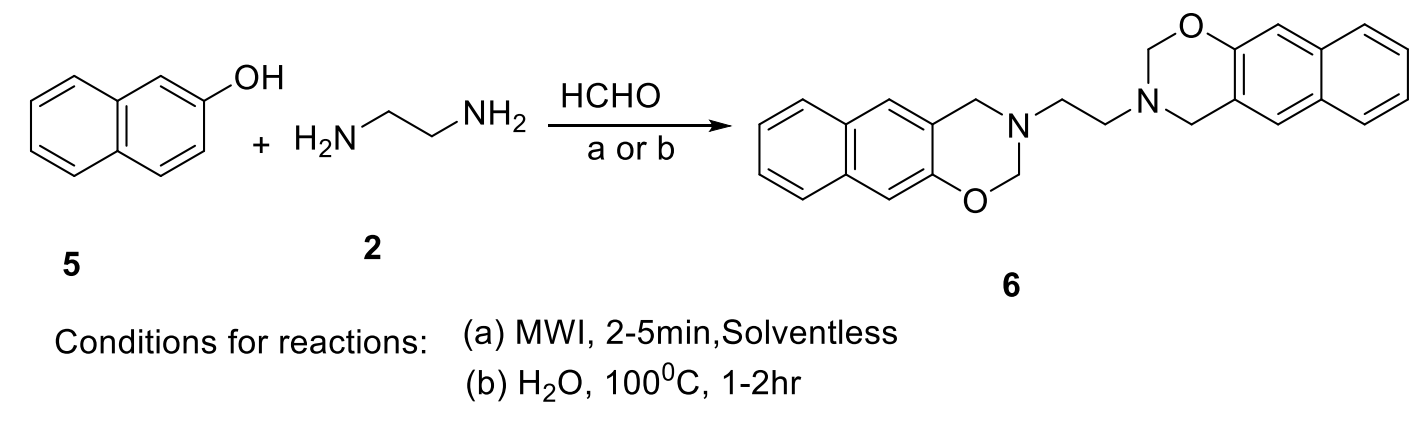

\section{Scheme 2}

Table I. Reaction time and yield under different conditions for compounds 4 a-j and 6 )

\begin{tabular}{|c|c|c|c|c|c|c|c|c|}
\hline \multicolumn{5}{|c|}{ Compound } & \multicolumn{2}{|c|}{$\begin{array}{l}\text { Microwave } \\
\text { Irradiation }\end{array}$} & \multicolumn{2}{|c|}{ Conventional Heating } \\
\hline Products & $\mathrm{R}^{1}$ & $\mathrm{R}^{2}$ & $\mathrm{R}^{3}$ & $x$ & $\begin{array}{l}\text { Yield } \\
(\%)\end{array}$ & $\begin{array}{l}\text { Reaction } \\
\text { time }(\mathrm{sec})\end{array}$ & Yield (\%) & $\begin{array}{l}\text { Reaction } \\
\text { time (hr) }\end{array}$ \\
\hline $4 a$ & $\mathrm{H}$ & $\mathrm{H}$ & $\mathrm{H}$ & $\mathrm{O}$ & 78 & 180 & 63 & 2 \\
\hline $4 b$ & $\mathrm{H}$ & $\mathrm{H}$ & $\mathrm{CH}_{3}$ & 0 & 76 & 180 & 58 & 2 \\
\hline $4 c$ & $\mathrm{CH}_{3}$ & $\mathrm{H}$ & $\mathrm{H}$ & $\mathrm{O}$ & 75 & 180 & 62 & 2 \\
\hline $4 d$ & $\mathrm{H}$ & $\mathrm{CH}_{3}$ & $\mathrm{H}$ & $\mathrm{O}$ & 78 & 180 & 60 & 2 \\
\hline $4 e$ & $\mathrm{C}_{6} \mathrm{H}_{5}$ & $\mathrm{H}$ & $\mathrm{H}$ & $\mathrm{O}$ & 75 & 180 & 59 & 2 \\
\hline $4 f$ & $\mathrm{H}$ & $\mathrm{C}_{6} \mathrm{H}_{5}$ & $\mathrm{H}$ & $\mathrm{O}$ & 76 & 180 & 61 & 2 \\
\hline $4 g$ & $\mathrm{H}$ & $\mathrm{H}$ & $\mathrm{OCH}_{3}$ & $\mathrm{O}$ & 76 & 180 & 58 & 2 \\
\hline $4 h$ & $\mathrm{H}$ & $\mathrm{H}$ & $\mathrm{H}$ & $\mathrm{S}$ & 80 & 120 & 65 & 1 \\
\hline $4 \mathbf{i}$ & $\mathrm{OCH}_{3}$ & $\mathrm{H}$ & $\mathrm{H}$ & $S$ & 82 & 120 & 68 & 1 \\
\hline $4 j$ & $\mathrm{H}$ & $\mathrm{OCH}_{3}$ & $\mathrm{H}$ & $S$ & 79 & 120 & 65 & 1 \\
\hline 6 & --- & ---- & $\mathrm{H}$ & 0 & 78 & 180 & 60 & 2 \\
\hline
\end{tabular}




\section{Conclusions}

Bisbenzoxazines and bis-benzothiazine exhibit high melting points compared to their monomers. ${ }^{26}$ Table 1 shows that reactions conventionally heating took longer to complete and produced a lower yield compared to the reactions under microwave irradiation. The difference in total reaction time and increased yields confirms the advantages of microwave asssited reactions compared to conventional heating.

\section{Experimental Section}

General. Microwave reactions were carried out in a CEM Discover Benchmate microwave digester. Melting points are recorded in open capillary tubes and are uncorrected. Infrared spectra were recorded on a BOMEM DA-8 FTIR instrument and the frequencies are expressed in $\mathrm{cm}^{-1} .{ }^{1} \mathrm{H}$ and ${ }^{13} \mathrm{C} \mathrm{NMR}$ spectra were recorded on a Bruker Avance II-400 spectrometer using $\mathrm{CDCl}_{3}$ as the solvent. Chemical shifts are reported in ppm downfield from internal tetramethylsilane and are given on the $\delta$ scale. Mass spectral data were obtained with a JEOL D300 (EI) mass spectrometer. Elemental analyses were carried out on a Heraeus CHN-O-Rapid analyzer. All compounds give satisfactory elemental analyses within $\pm 0.4 \%$ of the theoretical values. All reactions were monitored by TLC using precoated aluminum sheets (silica gel $60 \mathrm{~F}_{254} 0.2 \mathrm{~mm}$ thicknesses) and developed in an iodine chamber or under UVGL-15 mineral light 254 lamp. Column chromatographic separations were carried out using ACME silica gel (60-120 mesh).

Procedure for the preparation of the title compound (4a-j) by the conventional heating method. To a solution of 1 or $5(5 \mathrm{mmol})$ in water $(10 \mathrm{ml})$, formaldehyde (37\% by wt.) $(20 \mathrm{mmol})$ was added. A thick milky precipitate was formed. After $15 \mathrm{~min}$., diamine (2) $(10 \mathrm{mmol})$ was added and the reaction mixture was heated under reflux for 1-2 hrs. After reaction was completed (as monitored by TLC), the crude compound was extracted with ethyl acetate and purified by either by column chromatography using ethyl acetate/hexane as an eluent or by repeated recrystallisation from hot ethanol.

Procedure for the preparation of the title compounds (4a-j and 6) by microwave irradiation. To a mixture of 1 or $5(5 \mathrm{mmol})$, formaldehyde ( $37 \%$ by wt.) $(20 \mathrm{mmol})$ and $2(10 \mathrm{mmol})$ was added and the reaction mixtures were irradiated at 5 bar, $100 \mathrm{~W}, 100^{\circ} \mathrm{C}$ for specific times (Table-1). After reaction was complete (as monitored by TLC), the mixture was extracted with ethyl acetate and purified either by column chromatography using ethyl acetate/hexane or by repeated recrystallisation from hot ethanol.

1,2-Bis(2H-benzo[e][1,3]oxazin-3(4H)-yl)ethane (4a). Mp 107-09 ${ }^{\circ}$; IR (KBr): 2923, 2817, 1551, $1488 \mathrm{~cm}^{-1} ;{ }^{1} \mathrm{H}$ NMR $\left(\mathrm{CDCl}_{3}\right): \delta 2.47\left(\mathrm{~s}, 4 \mathrm{H},-\mathrm{CH}_{2}-\right), 3.46\left(\mathrm{~s}, 4 \mathrm{H}, \mathrm{CH}_{2}\right), 4.93\left(\mathrm{~s}, 4 \mathrm{H}, \mathrm{N}-\mathrm{CH}_{2}-\mathrm{O}\right), 6.78-7.15(\mathrm{~m}, 8 \mathrm{H}, \mathrm{ArH}) ;{ }^{13} \mathrm{C} \mathrm{NMR}$ $\left(\mathrm{CDCl}_{3}\right): \delta$ 49.55, 50.38, 84.24, 115.45, 120.53, 126.93, 128.32, 128.65, 158.12; Mass: $\mathrm{m} / z 297\left[\mathrm{M}^{+}+1\right]$; Anal. Calcd for $\mathrm{C}_{18} \mathrm{H}_{20} \mathrm{~N}_{2} \mathrm{O}_{2} ; \mathrm{C}, 72.95 ; \mathrm{H}, 6.80 ; \mathrm{N}, 9.45$; Found: C, 72.88; $\mathrm{H}, 6.67 ; \mathrm{N}, 9.54 \%$.

1,2-Bis(8-methyl-2H-benzo[e][1,3]oxazin-3(4H)-yl)ethane (4b). Mp: 108-110 ${ }^{\circ}$; IR (KBr, $\left.\mathrm{cm}^{-1}\right): 2945,2853$, 1208, 1043, 930, 857, 771; ${ }^{\mathrm{H}} \mathrm{NMR}\left(\mathrm{CDCl}_{3}\right): \delta 2.10\left(\mathrm{~s}, 6 \mathrm{H}, \mathrm{CH}_{3}\right), 2.93\left(\mathrm{~s}, 4 \mathrm{H},-\mathrm{CH}_{2}-\right), 3.99\left(\mathrm{~s}, 4 \mathrm{H}, \mathrm{CH}_{2}\right), 4.87(\mathrm{~s}, 4 \mathrm{H}$, $\left.\mathrm{N}-\mathrm{CH}_{2}-\mathrm{O}\right), 6.68-7.18(\mathrm{~m}, 6 \mathrm{H}, \mathrm{ArH}) ;{ }^{13} \mathrm{C} \mathrm{NMR}\left(\mathrm{CDCl}_{3}\right): \delta 14.57,48.33,49.42,81.56,118.00,118.96,124.00$, 124.58, 127.90, 151.07; Mass: $m / z 325\left[\mathrm{M}^{+}+1\right]$; Anal. Calcd for $\mathrm{C}_{20} \mathrm{H}_{24} \mathrm{~N}_{2} \mathrm{O}_{2}$ : C, 74.04; H, 7.46; N, 8.64; Found: C, 74.16; $\mathrm{H}, 7.37 ; \mathrm{N}, 8.71 \%$.

1,2-Bis(6-methyl-2H-benzo[e][1,3]oxazin-3(4H)-yl)ethane (4c). Mp: 109-111 ${ }^{\circ} \mathrm{C} ; \mathrm{IR}\left(\mathrm{KBr}, \mathrm{cm}^{-1}\right): 2936,2862$, 1200, 1100, 990, 900, 791; ${ }^{\mathrm{H}} \mathrm{NMR}\left(\mathrm{CDCl}_{3}\right): \delta 2.27$ (s, 6H, $\left.\mathrm{CH}_{3}\right), 2.98\left(\mathrm{~s}, 4 \mathrm{H},-\mathrm{CH}_{2}-\right), 3.98\left(\mathrm{~s}, 4 \mathrm{H}, \mathrm{CH}_{2}\right), 4.86(\mathrm{~s}, 4 \mathrm{H}$, 
$\left.\mathrm{N}-\mathrm{CH}_{2}-\mathrm{O}\right), 6.73-7.07(\mathrm{~m}, 6 \mathrm{H}, \mathrm{ArH}) ;{ }^{13} \mathrm{C} \mathrm{NMR}\left(\mathrm{CDCl}_{3}\right): \delta 17.85,49.13,50.85,82.58,115.70,119.43,126.32$, 130.71, 131.42, 153.95;Mass: $\mathrm{m} / \mathrm{z} 325$ [ $\left.\mathrm{M}^{+}+1\right]$; Anal. Calcd for $\mathrm{C}_{20} \mathrm{H}_{24} \mathrm{~N}_{2} \mathrm{O}_{2}$ : C, 74.04; H, 7.46; N, 8.64; Found: C, $\% 74.07 ; \mathrm{H}, 7.52 ; \mathrm{N}, 8.58 \%$.

1,2-Bis(7-methyl-2H-benzo[e][1,3]oxazin-3(4H)-yl)ethane (4d). Mp: 107-109 ${ }^{\circ}$; IR (KBr, $\left.\mathrm{cm}^{-1}\right): 2962,2847$, 1280, 1090, 989, 875, 790; ${ }^{1} \mathrm{H}$ NMR(CDCl 3$): \delta 2.22\left(\mathrm{~s}, 6 \mathrm{H}, \mathrm{CH}_{3}\right), 2.96\left(\mathrm{~s}, 4 \mathrm{H},-\mathrm{CH}_{2}-\right), 3.87\left(\mathrm{~s}, 4 \mathrm{H}, \mathrm{CH}_{2}\right), 4.93(\mathrm{~s}, 4 \mathrm{H}$, $\left.\mathrm{N}-\mathrm{CH}_{2}-\mathrm{O}\right), 6.73-6.97(\mathrm{~m}, 6 \mathrm{H}, \mathrm{ArH}) ;{ }^{13} \mathrm{C} \mathrm{NMR}\left(\mathrm{CDCl}_{3}\right): \delta$ 18.32, 49.03, 51.37, 82.11, 117.42, 121.61, 122.51, 128.92, 135.31, 156.23;Mass: $\mathrm{m} / \mathrm{z} 324$ [M+]; Anal. Calcd for $\mathrm{C}_{20} \mathrm{H}_{24} \mathrm{~N}_{2} \mathrm{O}_{2} ; \mathrm{C}, 74.04 ; \mathrm{H}, 7.46 ; \mathrm{N}, 8.64$; Found: C, $74.11 ; \mathrm{H}, 7.40 ; \mathrm{N}, 8.54 \%$.

1,2-Bis(6-phenyl-2H-benzo[e][1,3]oxazin-3(4H)-yl)ethane (4e). Mp: 102-103 ${ }^{\circ} \mathrm{C}$; IR (KBr, cm-1): 3011, 2894, 1250, 1100, 950, 890, 810; ${ }^{1} \mathrm{H} \mathrm{NMR}\left(\mathrm{CDCl}_{3}\right): \delta 2.95$ (s, 4H, $-\mathrm{CH}_{2}-$ ), $3.96\left(\mathrm{~s}, 4 \mathrm{H}, \mathrm{CH}_{2}\right), 4.98\left(\mathrm{~s}, 4 \mathrm{H}, \mathrm{N}-\mathrm{CH}_{2}-\mathrm{O}\right), 6.98-$ $7.46(\mathrm{~m}, 16 \mathrm{H}, \mathrm{ArH}) ;{ }^{13} \mathrm{C} \mathrm{NMR}\left(\mathrm{CDCl}_{3}\right): \delta 50.33,52.71,83.46,120.17,121.32,126.73,127.35,128.65,129.11$, 129.96, 134.82, 137.49, 154.44;Mass: $\mathrm{m} / \mathrm{z} 449\left[\mathrm{M}^{+}+1\right]$; Anal. Calcd for $\mathrm{C}_{30} \mathrm{H}_{28} \mathrm{~N}_{2} \mathrm{O}_{2}$ : C, 80.33; $\mathrm{H}, 6.29 ; \mathrm{N}, 6.25$; Found: $\mathrm{C}, 80.41 ; \mathrm{H}, 6.37 ; \mathrm{N}, 6.18 \%$.

1,2-Bis(7-phenyl-2H-benzo[e][1,3]oxazin-3(4H)-yl)ethane (4f). Mp: 101-103 ${ }^{\circ} \mathrm{C}$; IR (KBr, $\left.\mathrm{cm}^{-1}\right)$ : 2996, 2888, 1230, 1117, 960, 861, 791; ${ }^{1} \mathrm{H}$ NMR(CDCl 3$): \delta 2.95$ (s, 4H, $-\mathrm{CH}_{2}-$ ), $3.96\left(\mathrm{~s}, 4 \mathrm{H}, \mathrm{CH}_{2}\right), 4.86\left(\mathrm{~s}, 4 \mathrm{H}, \mathrm{N}-\mathrm{CH}_{2}-\mathrm{O}\right), 6.97-$ $7.42(\mathrm{~m}, 16 \mathrm{H}, \mathrm{ArH}) ;{ }^{13} \mathrm{C} \mathrm{NMR}\left(\mathrm{CDCl}_{3}\right): \delta 51.23,52.55,83.74,112.38,118.32,125.57,127.61,128.05,129.21$, 130.19, 139.94, 143.85, 156.28;Mass: $\mathrm{m} / \mathrm{z} 448$ [M+]; Anal. Calcd for $\mathrm{C}_{30} \mathrm{H}_{28} \mathrm{~N}_{2} \mathrm{O}_{2}$ : C, 80.33; $\mathrm{H}, 6.29 ; \mathrm{N}, 6.25$; Found: $\mathrm{C}, 80.38 ; \mathrm{H}, 6.21 ; \mathrm{N}, 6.30 \%$.

1,2-Bis(8-methoxy-2H-benzo[e][1,3]oxazin-3(4H)-yl)ethane (4g). Mp: 106-108 ${ }^{\circ} \mathrm{C}$; IR (KBr, $\left.\mathrm{cm}^{-1}\right): 2948,2822$, 1209, 1025, 925, 870, 717; ${ }^{\mathrm{H}} \mathrm{NMR}\left(\mathrm{CDCl}_{3}\right): \delta 2.91\left(\mathrm{~s}, 4 \mathrm{H},-\mathrm{CH}_{2}-\right), 3.96\left(\mathrm{~s}, 4 \mathrm{H}, \mathrm{CH}_{2}\right), 3.87(\mathrm{~s}, 6 \mathrm{H}, \mathrm{OCH}), 4.88(\mathrm{~s}$, $\left.4 \mathrm{H}, \mathrm{N}-\mathrm{CH}_{2}-\mathrm{O}\right), 6.86-7.02(\mathrm{~m}, 6 \mathrm{H}, \mathrm{ArH}) ;{ }^{13} \mathrm{C} \mathrm{NMR}\left(\mathrm{CDCl}_{3}\right): \delta 50.23,51.85,56.07,83.47,104.23,104.65,107.48$, 130.16, 156.28, 157.85; Mass: $\mathrm{m} / z 356\left[\mathrm{M}^{+}\right]$; Anal. Calcd for $\mathrm{C}_{20} \mathrm{H}_{24} \mathrm{~N}_{2} \mathrm{O}_{2}$ : C, 67.40; $\mathrm{H}, 6.79 ; \mathrm{N}, 7.86$; Found: C, $67.51 ; \mathrm{H}, 6.84 ; \mathrm{N}, 7.73 \%$.

1,2-Bis(2H-benzo[e][1,3]thiazin-3(4H)-yl)ethane (4h). Mp: 98-99 ${ }^{\circ} \mathrm{C}$; IR $\left(\mathrm{KBr}, \mathrm{cm}^{-1}\right)$ : 2948, 2822, 1210, 1090,

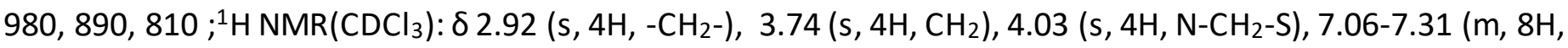
$\mathrm{ArH}) ;{ }^{13} \mathrm{C} \mathrm{NMR}\left(\mathrm{CDCl}_{3}\right): \delta 50.32,58.47,62.18,126.31,127.43,128.04,128.32,130.86,137.29 ;$ Mass: $\mathrm{m} / z 328$ $\left[\mathrm{M}^{+}\right]$; Anal. Calcd for $\mathrm{C}_{18} \mathrm{H}_{20} \mathrm{~N}_{2} \mathrm{~S}_{2}$ : C, 65.81; $\mathrm{H}, 6.14 ; \mathrm{N}, 8.53$; Found: $\mathrm{C}, 65.73 ; \mathrm{H}, 6.28 ; \mathrm{N}, 8.41 \%$.

1,2-Bis(6-methoxy-2H-benzo[e][1,3]thiazin-3(4H)-yl)ethane (4i). $\mathrm{Mp}$ : 95-98 ${ }^{\circ} \mathrm{C}$; IR (KBr, cm-1): 2985, 2864, 1220, 1101, 990, 815, $791 \mathrm{~cm}^{-1} ;{ }^{1} \mathrm{H} \mathrm{NMR}\left(\mathrm{CDCl}_{3}\right): \delta 2.93\left(\mathrm{~s}, 4 \mathrm{H},-\mathrm{CH}_{2}-\right), 3.73\left(\mathrm{~s}, 4 \mathrm{H}, \mathrm{CH}_{2}\right), 3.95(\mathrm{~s}, 6 \mathrm{H}, \mathrm{OCH}), 4.98$ $\left(\mathrm{s}, 4 \mathrm{H}, \mathrm{N}-\mathrm{CH}_{2}-\mathrm{S}\right), 6.98-7.22(\mathrm{~m}, 6 \mathrm{H}, \mathrm{ArH}) ;{ }^{13} \mathrm{C} \mathrm{NMR}\left(\mathrm{CDCl}_{3}\right): \delta 51.43,54.15,61.53,63.11,113.45,114.09,124.57$, 127.42, 132.65, 156.83;Mass: $\mathrm{m} / z 388$ [ $\left.\mathrm{M}^{+}\right]$; Anal. Calcd for $\mathrm{C}_{20} \mathrm{H}_{24} \mathrm{~N}_{2} \mathrm{O}_{2} \mathrm{~S}_{2}$ : C, 61.82; $\mathrm{H}, 6.23 ; \mathrm{N}, 7.21$; Found: C, $61.75 ; \mathrm{H}, 6.14 ; \mathrm{N}, 7.30 \%$.

1,2-Bis(7-methoxy-2H-benzo[e][1,3]thiazin-3(4H)-yl)ethane (4j). Mp: 99-102 ${ }^{\circ} \mathrm{C}$; IR (KBr, $\left.\mathrm{cm}^{-1}\right)$ : 2977, 2859, 1205, 1041, 935, 860, 765; ${ }^{\mathrm{H}} \mathrm{NMR}\left(\mathrm{CDCl}_{3}\right): \delta 2.92\left(\mathrm{~s}, 4 \mathrm{H},-\mathrm{CH}_{2}-\right), 3.78\left(\mathrm{~s}, 4 \mathrm{H}, \mathrm{CH}_{2}\right), 3.91\left(\mathrm{~s}, 6 \mathrm{H}, \mathrm{OCH}_{3}\right), 4.03(\mathrm{~s}$,

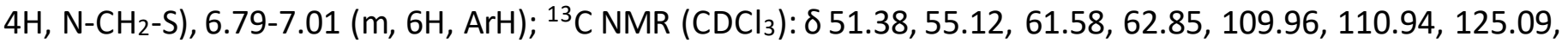
130.64, 132.75, 157.68;Mass: $m / z 389\left[\mathrm{M}^{+}+1\right]$; Anal. Calcd for $\mathrm{C}_{20} \mathrm{H}_{24} \mathrm{~N}_{2} \mathrm{O}_{2} \mathrm{~S} 2: \mathrm{C}, 61.82 ; \mathrm{H}, 6.23 ; \mathrm{N}, 7.21$; Found: C, $61.72 ; \mathrm{H}, 6.34 ; \mathrm{N}, 7.17 \%$.

1,2-Bis(2H-naphtho[2,3-e][1,3]oxazin-3(4H)-yl)ethane (6). Mp: 108-110 ${ }^{\circ}$; IR (KBr, $\left.\mathrm{cm}^{-1}\right): 2967,2851,1210$, 1061, 993, 875, 792; ${ }^{1} \mathrm{H} N M R\left(\mathrm{CDCl}_{3}\right): \delta 2.93$ (s, 4H, - $\left.\mathrm{CH}_{2}-\right)$, $3.99\left(\mathrm{~s}, 4 \mathrm{H}, \mathrm{CH}_{2}\right), 5.03\left(\mathrm{~s}, 4 \mathrm{H}, \mathrm{N}-\mathrm{CH}_{2}-\mathrm{O}\right), 7.35-7.81(\mathrm{~m}$, $12 \mathrm{H}, \mathrm{ArH}) ;{ }^{13} \mathrm{C} \mathrm{NMR}\left(\mathrm{CDCl}_{3}\right): \delta 50.92$, , 52.33, 83.17, 107.48, 122.52, 125.74, 126.28, 127.54, 128.36, 128.96, 129.42, 132.66,157.43;Mass: $\mathrm{m} / \mathrm{z} 396$ [M+]; Anal. Calcd for $\mathrm{C}_{26} \mathrm{H}_{24} \mathrm{~N}_{2} \mathrm{O}_{2}$ : C, 78.76; H, 6.10; N, 7.07; Found: C, $78.83 ; \mathrm{H}, 6.22 ; \mathrm{N}, 7.15 \%$. 


\section{Acknowledgements}

We gratefully acknowledge financial support from SERB-DST, New Delhi, India for Financial support and SAIFNEHU, Shillong, India for the spectral analysis.

\section{References}

1. Takimoto, H.; Calvo E. Principles of Oncologic Pharmacotherapy, Pazdur, R. ; Wagman L. D. ; Camphausen K. A. ; Hoskins, W. J. Eds. Cancer Management: A Multidisciplinary Approach. 11th Edition, 2008. (a) Ohnacker, G. ; Scheffler, H. US Pat. 2943 087, 1960.

2. Reichert, B. Die Mannich Reaction; Springer-Verlag: Berlin, 1959. https://doi.org/10.1007/978-3-642-86313-4

3. Kuehne, M. E.; Konopke, E. A. J. Med. Chem. 1962, 5, 257. https://doi.org/10.1021/jm01237a005

4. Chylinska, J. B.; Janowiec, M.; Urbanski, T. Br. J. Pharmacol. 1971, 43, 649. https://doi.org/10.1111/j.1476-5381.1971.tb07194.x

5. Pedersen, O. S.; Pedersen, E. B. Synthesis 2000, 479. https://doi.org/10.1055/s-2000-6357

6. Duffin, W. M.; Rollo, I. M. Br. J. Pharmacol. 1957, 12, 171. https://doi.org/10.1111/j.1476-5381.1957.tb00116.x

7. Singh, O. V.; Han, H. Tetrahedron Lett. 2007, 48, 2345. https://doi.org/10.1016/j.tetlet.2007.01.145

8. Joyce, J. N.; Presgraves, S.; Renish, L.; Borwege, S.; Osredkar, D. H.; Replogle, M.; PazSoldan, M.; Millan, M. J. Exp. Neurol. 2003, 184, 393.

9. Abe, K.; Yammamoto, S.; Matsui, K. Yakagaku Zasshi 1956, 76, 1058. https://doi.org/10.1248/yakushi1947.76.9 1058

10. Lombardino, J. G.; Wiseman, E. H.; Mclamore, W. J. Med. Chem. 1971, 14, 117.

11. Rehman, M. Z.; Anwar, J.; Ahmad, S. Bull. Korean. Chem. Soc. 2005,26, 1771.

12. Turck, D.; Busch, U.; Heinzel, G.; Narjes, H.; Nehmiz, G. J. Clin. Pharmacol. 1996, 36 ,79. https://doi.org/10.1002/j.1552-4604.1996.tb04155.x

13. Boschail, C.; Cana, A.; Disfilo, R.; Frutlero, A.; Gasco Bioorg. Med. Chem. 2000, 7, 1727. https://doi.org/10.1016/S0968-0896(00)00098-5

14. Clark, R. D.; Carron, J. M.; Kloge, A. F.; Repke, D. B.; Roszkowski, A. P.; Strosberg, A. M.; Earkar, S. B.; Bitter,S. M.; Okando, M. D. J. Med. Chem. 1983, 26, 657.

15. Rajanarendar, E.; Mohan, G. A. S. J. Chem. 2008, 47B, 112.

16. Bhatia, S. H.; Buckley, D. M.; McCabe, R. W.; Avent, A.; Brown, R. G.; Hitchcock, P. B. J. Chem. Soc., Perkin Trans. 1998, 1, 569. https://doi.org/10.1039/a705331b

17. Grenda, V. J.; Jones, R. E.; Gal. G.; Sletzinger, M. J. Org. Chem. 1965, 30, 259. https://doi.org/10.1021/jo01012a061

18. Kerns, R. J.; Rybak, M. J.; Kaatz, G. W.; Vaka, F.; Cha, R.; Grucz, R. G.; Diwadkar,V. U.; Ward, T. D. Bioorg. Med. Chem. Lett. 2003, 13, 1745. https://doi.org/10.1016/S0960-894X(03)00208-7 
19. Posner, G. H.; Ploypradith, P.; Parker, M. H.; O’Dowd, H.; Woo, S. H.; Northrop, J.; Krasavin, M.; Dolan, P.; Kensler, T. W.; Xie. S.; Shapiro, T. A. J. Med. Chem. 1999, 42, 4275.

https://doi.org/10.1021/jm990363d

20. Carlier, P. R.; Han, Y.; Chow, E. H.; Li, C. P. -L.; Wang, H.; Lieu,T. X.; Wong.H. S.; Pang,Y. P. Bioorg. Med. Chem.Lett. 1999, 7, 351.

https://doi.org/10.1016/S0968-0896(98)00213-2

21. Kumar, K. S. S.; Nair,C. P. R.; Radhakrishnan,T. S.; Ninan, K. N. Eur. Polymer Jour. 2007, 43, 2504.

https://doi.org/10.1016/i.eurpolymj.2007.03.028

22. Pei, D. J.; Gu, Y.; Cai, X. Acta Polym Sin (Chin) 1998, 5, 595.

23. Manikannan, R.; Muthusubramanian, S. Ind. Jour. Chem. 2010, 48B, 1083.

24. Szabo', J.; Szu^ cS, E.; Fodor, L.; Berna’ th, G.; Soha'r, P. Tetrahedron 1989, 45, 2731.

25. Jacob, D.; Meier, H. J. Heterocycl. Chem. 1986, 23, 1085.

https://doi.org/10.1002/jhet.5570230424

26. Augusto R.; Edgar O.; Armando S.; Pedro J. N. Heterocycles 1986, 24, 2507.

27. Burke, W. J.; Stephens, C. W. J. Am. Chem. Soc. 1952, 74, 1518.

https://doi.org/10.1021/ja01126a050

28. Palmieri, G. Eur. J. Org. Chem 1999, 64, 805. https://doi.org/10.1002/(SICI)1099-0690(199904)1999:4<805::AID-EJOC805>3.3.CO;2-I

29. Campi, E. M.; Jackson, W. R.; McCubbin, Q. J.; Trnacek, A. E. J. Chem. Soc., Chem. Commun. $1994,2763$. https://doi.org/10.1039/c39940002763

30. Vilkas, M.; Makani, S.; Mafroud, A. E. K. C. R. Acad. Sci. Ser. 2 1988, 307, 1851; Chem. Abstr. 1989, 111, 7311w.

31. Colin, J. L.; Loubinoux, B. Tetrahedron Lett. 1982, 23, 4245.

https://doi.org/10.1016/S0040-4039(00)88715-8

32. Aversa, M. C.; Giannetto, P.; Caristi, C.; Ferlazzo, A. J. Chem. Soc., Chem. Commun. 1982, 469.

33. Katritzky, A. R.; Xu, Y. J.; Jain, R. J. Org. Chem. 2002, 67 (23), 8234.

https://doi.org/10.1021/jo020336m

34. Burke, W. J. J. Am. Chem. Soc. 1949, 71, 609.

https://doi.org/10.1021/ja01170a063

35. Burke, W. J.; Kolbezen , M. J.; C.W. Stephens, M. J. J. Am. Chem. Soc. 1952, 74, 3601.

https://doi.org/10.1021/ja01134a039

36. Burke, W. J.; Murdock, K. C. ; Ec, G. J. Am. Chem. Soc. 1954, 76, 1677.

https://doi.org/10.1021/ja01635a065

37. Burke, W. J.; Reynolds, R. J. J. Am. Chem. Soc. 1954, 76, 1291.

https://doi.org/10.1021/ja01634a027

38. Burke,W. J.; Hammer, C.R.; Weatherbee, C. J. Org. Chem. 1961, 26, 4403.

https://doi.org/10.1021/jo01069a053

39. Fields, D. L.; Miller, J. B.; Reynolds, D. D. J. Org. Chem. 1962, 27, 2749.

https://doi.org/10.1021/jo01055a011

40. Rivera, A.; Gallo, G. I.; Gayon, M. E. Synth. Commun. 1994, 24, 2081.

41. Schmidt, C.; Thondorf, I.; Kolehmainen, E.; Bohmer, V.; Vogt, W.; Rissanen, K. Tetrahedron Lett. 1998, 39, 8833.

https://doi.org/10.1016/S0040-4039(98)02046-2

42. Higham, C. S.; Dowling, D. P.; Shaw, J. L.; Ziegler, J.; Farrell, J. R. Tetrahedron Lett. 2006, 47, 4419. 
https://doi.org/10.1016/j.tetlet.2006.04.077

43. Agag,T.; Takeichi, T. J. Polym. Sci. A Polym. Chem. 2007, 45, 1878.

https://doi.org/10.1002/pola.21953

44. Katritzky, A. R. ; Xu, Y. J.; Jain, R. J. Org. Chem. 2002, 67, 8234. https://doi.org/10.1021/jo020336m

45. Kiskan, B. ; Yagci, Y.; Sahmetlioglu, E.; Toppare, L. J. Polym. Sci. A Polym. Chem. 2007, 45, 999. https://doi.org/10.1002/pola.21848

46. Brunovska, Z.; Liu, J.P. ; Ishida, H. Macromol. Chem. Phys. 1999, 200, 1745. https://doi.org/10.1002/(SICI)1521-3935(19990701)200:7<1745::AID-MACP1745>3.0.CO;2-D

47. Allen, D. J.; Ishida, H. Polymer 2007, 48, 6763. https://doi.org/10.1016/j.polymer.2007.09.003

48. Dunkers, J.; Ishida, H. Spectrochim Acta 1995, 51A, 1061. 